\title{
ECG Rhythm Disturbances or Artefacts-Unusual Cause as Culprit
}

\author{
Mohit Kumar, Bhavna Gupta* and Sumedha Suresh \\ Assistant Professor, Department of Anesthesiology, All India Institute of Medical Sciences, Rishikesh
}

*Corresponding author: Bhavna Gupta, Assistant Professor, Department of anesthesiology, All India Institute of Medical Sciences (AIIMS), Rishikesh. Uttrakhand, India.

\section{Case Report}

A 15 year old American Society of Anaesthesiologist class 1 male, was, posted for modified radical mastoidectomy under general anaesthesia. On the day of the surgery, routine standard monitors were attached. The ECG on the monitor showed normal sinus rhythm with a heart rate of $88 / \mathrm{min}$, blood pressure of $100 / 70 \mathrm{mmHg}$, peripheral saturation of $99 \%$ on room air.He was pre-medicated with $1 \mathrm{mg}$ intravenous Midazolam, 80 micrograms fentanyl and induced with $100 \mathrm{mg}$ propofol and vecuronium 5 mg.Trachea was intubated with 6 mmID cuffed endotracheal tube and connected to anaesthesia workstation. Anaesthesia was maintained on 50:50 $\mathrm{O}_{2}: \mathrm{N}_{2} \mathrm{O}$ and Sevoflurane. As per routine norm, the patient was covered with a warming blanket (Geratherm medical warming system). A glance at the monitor revealed ECG changes suggestive of broad QRS complexes in lead $\mathbb{I}$, although blood pressure and peripheral saturation were within normal limits. We shifted the monitor to 6 lead ECG monitor, and we found the same ECG changes in lead I, I , aVF, aVR and aVL. The ECG showed broad QRS complex and changes mimicking those of bundle branch block (Figure 1). The patient was hemodynamically stable, there were no missed beats on radial pulse palpation and no variation in rhythm on the plethysmograph was observed.
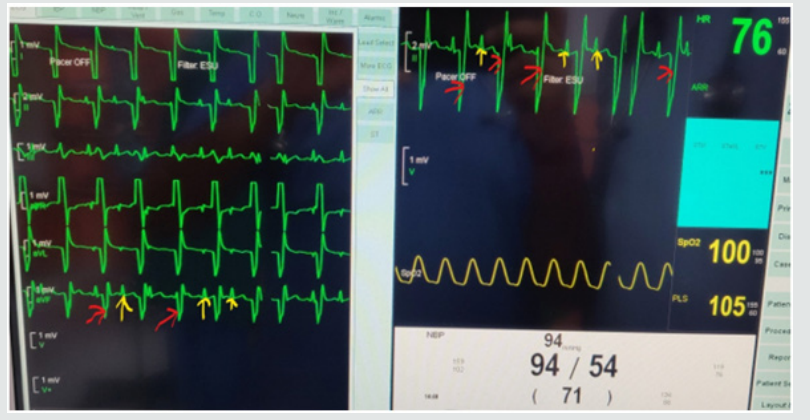

Figure 1: The ECG showed broad QRS complex and changes mimicking that of bundle branch block.
Meanwhile, we ruled out all the possible causes of arrhythmia-, there was no hypoxia, hypercarbia, pain, sympathetic stimulation. Arterial blood gases report was $\mathrm{pH} 7.39, \mathrm{PaO}_{2}-250, \mathrm{PaCO}_{2} 36, \mathrm{Na}$ $139, \mathrm{~K} 4.6, \mathrm{HCO}_{3} 22$. We further investigated and diagnosed this event as an ECG artefact. We went to look for the causes of the artefact which included-first checking the ECG leads and electrode for any disconnection and any electric breach. No external electric interference was observed. Anaesthesia workstation and OR table were checked for any earthing problem. A faulty monitor as the cause was ruled out as another monitor showed the same. We removed the possible causes of interferences such as mobile phones, cautery disturbances etc. During our search, we found that the warming blanket was connected to Geratherm medical warming electric system. To rule out any electric interference by this warming system, we turned off the system and found that the ECG of patient reverted to normal as in the preoperative period. To confirm that the artefact was caused by warming blanket system, we turned on Geratherm warming system again and we found the same ECG artefact on the monitor immediately.While perioperative ECG changes are a cause for concern to the anaesthesiologist, it is of utmost importance that the artefacts in the ECG be distinguished from the pathological variations as misinterpretations of the ECG can result in the patient being subjected to either unwanted investigations or treatment. Although in most of the cases, the artefact can quite easily be recognised as one, it is possible for it to mimic a more serious pathologic condition which requires immediate intervention and herein lies the importance of distinguishing a true pathology from an artefact. A history revealing no previous cardiac illness or symptoms, stable hemodynamics in the presence of the ECG changes, a peripheral pulse correlating with the apical pulse indicate that the ECG changes were artefacts and not arrhythmias. 
In this case, the patients's warming system that was Geratherm warming system, had caused electrical interference in the ECG, mimicking serious ECG changes. Our operation theatres have complete grounding electric and earthing system to minimize electrical interference despite which the warming system produced the changes in the ECG [1]. In an operating room environment, the patient is likely to be monitored by more than one device showing the heart rate and rhythm- the plethysmograph, the direct arterial blood pressure monitor and the CVP monitor. In the event of a true arrhythmia, changes in the rate and rhythm are likely to show up in all the monitors and not just the ECG. The presence of a normal plethysmograph during the electrocardiographic changes should arouse the suspicion that the changes are not pathologic. Also, pseudo arrhythmias are not responsive to the treatment given for the arrhythmia that they mimic. This lack of response to treatment, if administered, points towards the changes in the electrocardiogram being artefacts. The reversal to sinus rhythm on removal of the suspected source of the artefacts also indicates the absence of a pathology.

\section{Conclusion}

The consequences of falsely diagnosed arrhythmias may vary from treatment with anti-arrhythmic medication to the delivery of shocks by a defibrillator or even, the placement of a permanent pacemaker. It is thus crucial that the anaesthesiologist has a thorough knowledge of not only the pathology causing ECG variations but also the various artefacts that may be present.

\section{References}

1. Misra G, Dhiraaj S, Kapoor A, Goyal P (2018) An Unusual Cause of Electrocardiographic Artefact: A Patient's Warming Blanket. Turkish journal of anaesthesio1ogy and reanimation 46(2):161.

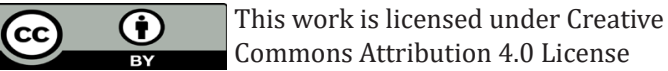

To Submit Your Article Click Here:

Submit Article

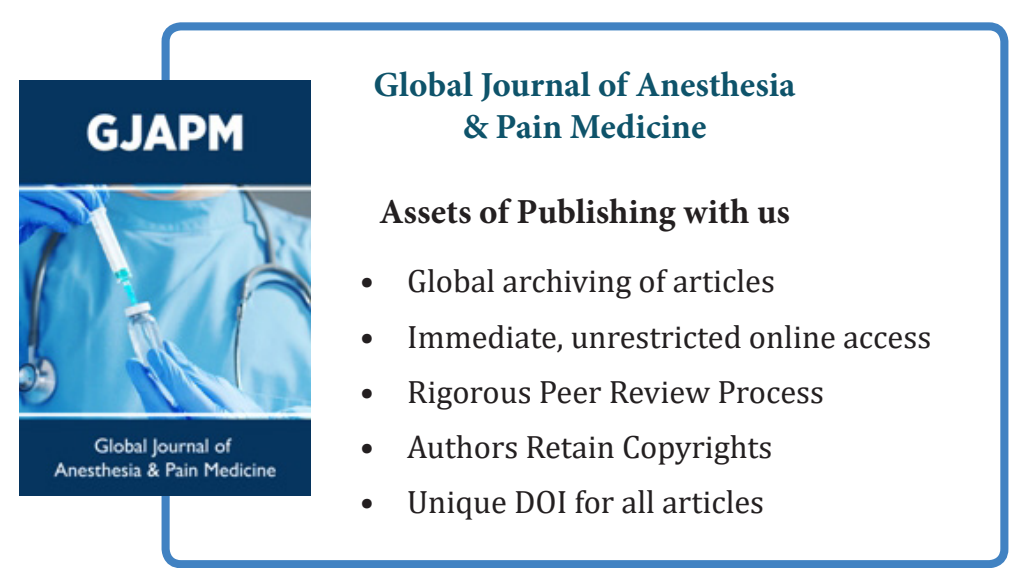

